\title{
EL OTRO MORISCO: ALGUNAS REFLEXIONES SOBRE EL ESTUDIO DE LA RELIGIOSIDAD MORISCA A TRAVÉS DE FUENTES NOTARIALES
}

\author{
Amalia García Pedraza*
}

Sugestivo como quizás ningún otro episodio de nuestra Historia Moderna, La vida y tragedia de los moriscos es, en la actualidad, una de las cuestiones historiográficas más debatidas. Lejos ya de los planteamientos positivistas con los que inicialmente se abordó su investigación, ha quedado también superado el enfoque que, bajo la influencia de Annales, interpretó la relación moriscos-cristianos como un enfrentamiento entre civilizaciones. Las obras aparecidas en las dos últimas décadas muestran el inicio de una revisión de todo cuanto representa el ser morisco. Se concibe ahora el estudio de esta minoría como el de una realidad cultural, económica y religiosa de primera magnitud y gran complejidad, a la que es necesario analizar a partir de las más variadas premisas conceptuales y metodológicas.

En este nuevo tratamiento del problema morisco ha sido fundamental la explotación sistemática de los fondos inquisitoriales, el estudio de la literatura aljamiada, y las incursiones cada vez más asiduas en los archivos notariales. Sin embargo, y a pesar del progreso experimentado, siguen subsistiendo lo que, retomando la expresión de Louis Cardaillac ${ }^{1}$, podríamos denominar "zonas de penumbra". Penumbra geográfica referente a espacios como Aragón, Las Baleares, Cataluña, Castilla la Vieja, que siguen presentando por comparación a otros ámbitos como Granada, Valencia, Toledo, un balance bibliográfico aún deficitario. Penumbra cronológica al ser principalmente tres eventos los que han centrado el interés de los historiadores: Conversión (1500-1501), Guerra de Granada (1568-1570), Expulsión (16091610). Finalmente penumbra temática. Esta última nos impide aún conocer muchos aspectos de su vida cotidiana, su estructura social y económica, su cultura material y un largo etcétera al que tenemos que incorporar, por para-

* Universidad de Granada.

1. Cardaillac, L. (dir.): Introducción a Les Morisques et l'Inquisition, París, 1990. 
dójico que nos pueda parecer, la propia religiosidad. Desconocimiento que calificamos como paradójico, más que por el volumen de publicaciones dedicadas exclusivamente a su estudio, por el hecho de que la mayoría de "les historiens du problème morisque ont abordé cette question d'une maniére ou d'une autre"

Se impone por tanto una reflexión sobre la manera que hasta ahora se ha tenido de abordar el fenómeno religioso, lo que implica en cierta medida hacerlo sobre la trayectoria de la propia Moriscología. Ésta, como afirmó Epalza $^{3}$, corre el peligro de encerrarse en un territorio en demasía aislado. La utilización de numerosa documentación de archivo y fuentes literarias no es suficiente si no viene acompañada de un esfuerzo por buscar nuevos marcos interpretativos, nuevos enfoques desde los que analizar el problema. Existe, como muy bien señala García-Arenal ${ }^{4}$, timidez interpretativa, que desde mi punto de vista queda patente sobre todo en los estudios de religiosidad.

Desde que Longás escribiera la única monografía consagrada a la religiosidad morisca ${ }^{5}$, apenas se han producido renovaciones en su línea de investigación. Todos los trabajos posteriores han partido de una idea apriorística: la vida religiosa de este grupo fue semejante, en lo esencial, a aquella de los pueblos cuya fe era el Islam. Su religiosidad consistió en una conversión nominal al cristianismo y una observancia de su fe ancestral. Queda así definido el morisco como un "moro encubierto", hereje secreto en el que sobraba el bautismo y faltaba la fe. Pero esta aseveración, con un fondo de verdad que no podemos negar, se fundamenta exclusivamente en la información ofrecida por las fuentes consultadas, que no han sido hasta ahora otras que las oficiales, las inquisitoriales y los tratados de polémica. Si bien es verdad que gracias a ellas se ha avanzado considerablemente en el conocimiento de la minoría morisca, no es menos cierto que lo hemos hecho en una única dirección: la que percibe la diferencia, la inasimilación y el conflicto. Como ha puesto de relieve Márquez Villanueva ${ }^{6}$, hemos construido la historia de este colectivo en base a fuentes del más puro carácter oficial, originadas desde las orillas más radicales y opuestas a la minoría. Hemos considerado entre los moriscos sólo la voz de los sectores disidentes, y de aquellos que estaban en circunstancias extremas, como es el caso de los acusados ante el Santo Oficio.

2. SABAGH, L.: "La religion des morisques entre deux Fatwas», Les Morisques et l'Inquisition..., p. 45.

3. Epalza, M.: Los moriscos antes y después de la Expulsión, Madrid, 1992, p. 18.

4. GARCía-ARENAL, M.: «El problema morisco: propuestas de discusión», Al-Qantara, 13-2 (1992), pp. 491-503.

5. LONGÁS, P.: Vida religiosa de los moriscos, Granada, 1991.

6. MÁRQuez Villanueva, F.: El problema morisco (desde otras laderas), Madrid, 1991. 
Pero un fenómeno tan complejo como el religioso no puede ser definido de manera tan parcial. Creo que hasta ahora hemos identificado el estudio de la religiosidad del morisco con la elaboración de un inventario de sus prácticas musulmanas, al que le hemos adjuntado un listado de las medidas tomadas para acabar con ellas. Con esta simplificación reducimos a un único patrón de religiosidad a toda una comunidad, obviando cualquier diferencia entre sus componentes. Pero ¿Qué ocurrió con los conversos sinceros?, ¿Cómo interiorizaron el mensaje cristiano?, ¿cómo entendieron y plasmaron los moriscos en las prácticas sociales los ritos y creencias de su nueva fe? ¿Qué relación hay entre el status socio-económico de sus miembros y la religiosidad manifestada por estos?, ¿no existió ninguna evolución en su religiosidad a lo largo de su permanencia en el territorio español?

No se puede estudiar la religiosidad de un pueblo sin contar con su propia sensibilidad y es precisamente eso lo que hemos obviado en nuestro estudio de los moriscos. Es necesario escuchar la voz del morisco, pero la voz emitida desde la normalidad ${ }^{7}$. Para reconstruir la religiosidad de la Europa cristiana no sólo hemos consultado fuentes oficiales e inquisitoriales, sino que nos hemos esforzado en buscar otras vías, ¿por qué no hacer lo mismo con los moriscos? Quizás tendríamos que dejar de cuestionarnos exclusivamente si llegaron o no a ser "verdaderos cristianos de nación" guntarnos como propone García-Arenal ¿Qué fue en realidad ser morisco?, ¿cuáles fueron los ingredientes de su identidad, y qué papel jugó la religión en la formación de la misma?

A estas preguntas sólo podremos responder si somos capaces de incorporar planteamientos interdisciplinares e instrumentos de investigación procedentes de otras Ciencias Sociales. Especialmente puede ser muy enriquecedora la colaboración con la antropología, al enmarcarse el problema morisco en la relatividad antropológica del concepto de asimilación. Pero me parece especialmente necesario aprovechar las inmensas posibilidades que para el

7. En esta línea de investigación es interesante el trabajo de La Parra. Escribe este historiador que "puede resultar clarificador, y mucho más coherente, enfocar la cuestión a partir de una doble diferenciación de planos: el «institucional y secular de la represión», por una parte, del «local y cotidiano», por otra, en el seno de la cual nosotros sí creemos que se da una verdadera convivencia". Sin entrar en si existió o no en el caso granadino esta convivencia, comparto el enfoque que propone, aunque yo subrayaría la necesidad de estudiar también la dialéctica entre ambos planos. LA PARRA LóPEZ, S.: «Moros y cristianos en la vida cotidiana: ¿historia de una represión sistemática o de una convivencia frustrada?», Revista de $\mathrm{H}^{a}$ Moderna. Anales de la Universidad de Alicante, 11 (1992), p. 148.

8. Sobre la cuestión de si llegaron a ser o no verdaderos cristianos de nación me remito a lo que expuse en «El morisco ante la muerte. Algunas reflexiones sobre los testamentos otorgados por los moriscos granadinos (1500-1526)", Homenaje a Louis Cardaillac (en prensa). En la actualidad me parece necesario una revisión de este planteamiento. Lo importante no es conocer si llegaron a asumir el modelo de cristiano que se les propuso sino su relación con el mismo, y el juego de reciprocidades existente entre la fe predicada y la fe asumida y vivida. 
estudio de la religiosidad nos ofrecen las escrituras notariales, como ya lo han demostrado numerosos trabajos inscritos dentro del campo de las mentalidades. Y es precisamente en esta última cuestión en la que me voy a centrar para reflexionar, brevemente, sobre la religiosidad del morisco granadino.

Pocos son los trabajos que, específicos del Reino granadino o referentes a otras zonas ${ }^{9}$, no se hayan hecho eco de la problemática de uno de los contingentes de cristianos nuevos más numerosos de nuestro país. Sin entrar en un estudio crítico de los mismos, sus investigaciones nos han servido para reconstruir aspectos imprescindibles en la comprensión de su religiosidad. En primer lugar hemos podido establecer cuáles fueron las obligaciones religiosas observadas por los moriscos que permanecieron fieles al Islam. Prácticas que debieron estar muy extendidas en los primeros años de la conversión, pero cuya evolución a lo largo del tiempo desconocemos ${ }^{10}$. En segundo lugar conocemos los cauces por los que discurrió la política asimiladora desplegada por las autoridades civiles y eclesiásticas: desde las conversiones ${ }^{11}$, hasta la organización de la Iglesia

9. Casi todos los trabajos sobre los moriscos de otros ámbitos geográficos se ocupan, en mayor o menor medida, de "los granadinos", es decir de los moriscos que tras la revuelta de la Alpujarras fueron dispersados por Castilla. La visión que de este contingente se nos ofrece en estos estudios puede quedar ejemplificada a través de la dada por Juan Bautista Vilar, quien dice: "los de Granada sabían ocultar su convicciones y prácticas clandestinas mejor que sus rudos correligionarios murcianos. Eran más instruidos que estos, y por lo mismo más impermeables a la evangelización". VILAR, J. B.: Los moriscos del reino de Murcia y Obispado de Orihuela, Murcia, 1992, p. 74. Referencias al colectivo granadino fuera de su territorio encontramos, entre otras, en las obras de: BENÍTEZ SÁNCHEZ-BLANCO, R.: «Un plan para la aculturación de los moriscos valencianos: "las ordenaciones de Ramírez de Haro. 1540"», Les morisques et leur temps, París, 1990, pp. 127-157. DEDIEU, J. P.: «Entre religión y política: los moriscos», Revista d'Historia Moderna. Manuscris, 12 (1994), pp. 63-78. FERNÁNDEZ NIEVA, J.: «Inquisición y minorías Etnico-religiosas en Extremadura», Revista de Estudios Extremeños, LXI (1985), pp. 213-260. TAPIA SÁNCHEZ, S. de: La comunidad morisca de Ávila, Salamanca, 1991.

10. Junto a la mencionada obra de Longás, quien reconstruye las obligaciones religiosas de este colectivo en base a los cinco preceptos del Islam, se puede recopilar más información al respecto en obras como la de Gallego y Burín y GAMIR SANDOVAL, A.: Los moriscos del Reino de Granada según el Sinodo de Guadix en 1554, Granada, 1968. También en dos obras que podríamos calificar de clásicas como son las de CARO BAROJA, J.: Los moriscos del Reino de Granada, Madrid, 1985 y DOMínguez ORTIZ, A. y VINCENT, B.: Historia de los moriscos. Vida y tragedia de una minoría, Madrid, 1989. Para terminar diremos que uno de los trabajos más recientes dedicados exclusivamente a la religiosidad morisca vuelve a reincidir sobre los mismos aspectos, nos referimos al artículo de CABANELAS RODRIGUEZ, D.: «Los moriscos: vida religiosa y evangelización», La incorporación de Granada a la Corona de Castilla, Granada, 1993, pp. 497-511.

11. Entre los historiadores que se han ocupado de esta temática queremos subrayar aquí los trabajos de GALÁN SÁNCHEZ, A.: «Notas para una periodización de la historia de los moriscos granadinos. De las capitulaciones de la conversión a las medidas de la Capilla Real», Actas del III Coloquio de Historia Medieval Andaluza, Jaén, 1984. López DE COCA CASTAÑER, J. E.: «La conversión General en el obispado de Málaga (1500-1501)», Chronica Nova, 21 (19931994), pp. 191-237. 
granadina y su labor evangelizadora ${ }^{12}$, pasando por la actuación del Santo Oficio ${ }^{13}$.

De todos estos trabajos, sea cual sea el objetivo en el que se centren, se desprende una concepción de la religiosidad morisca que podríamos sintetizar con las palabras de uno de los máximos especialistas en la historia de este grupo: "Los granadinos conservaron hasta el momento de la expulsión su dialecto árabe, observaron los preceptos coránicos, preservaron los ritos que acompañaban a los nacimientos, matrimonios o entierros... A pesar de todo un arsenal de textos normativos conducentes a la asimilación, de las campañas de evangelización y de una represión multiforme, los moriscos se aferraban a su fe y constituían un bloque prácticamente intacto"14.

Pero esta concepción del morisco granadino está en fase de revisión. En primer lugar el "bloque" morisco no fue tan compacto ni tan uniforme. En el caso de la nobleza nazarí tenemos una asimilación rápida, que permitió a la corona utilizar a destacados miembros de la misma como enlace entre ella y el pueblo convertido ${ }^{15}$. Moriscos "colaboracionistas", incorporados a la élite

12. La estrategia que siguió corona e iglesia en la conversión de estos "infieles" ha sido analizada al tratar puntos como la organización de la iglesia granadina y la dotación de sus parroquias, la fundación de colegios destinados al adoctrinamiento de moriscos, la labor desempeñada por algunos de sus prelados (especialmente estudiadas están las figuras de Talavera y Guerrero), el parecer de teólogos y juristas sobre el estado de los cristianos nuevos de este reino...etc. Citaremos aquí por cuestiones de espacio algunos de los trabajos que nos parecen más ilustrativos: Álvarez RoDríguez, J. R.: «La casa de doctrina del Albaicín. Labor apostólica de la Compañía de Jesús con los moriscos», CA, 19-20 (1983-1984), pp. 233-246. AzCONA, T. de: «Diversos criterios sobre la conversión de los moros de Granada», XX Siglos, I, 3-4 (1990), pp. 139-150. FERNÁNDEZ DE MADRID, A.: Vida de Fray Hernado de Talavera, primer arzobispo de Granada, Edición, est. y notas por el P.F.G. Olmo (1931), ed. facsímil, estudio preliminar de Martínez MedinA, F.G, Granada, 1992. Garkido ARRANDA, A. «Papel de la Iglesia de Granada en la asimilación de la Sociedad morisca», Anuario de Historia Moderna y Contemporánea, 1-3 (1974-1976), pp. 70-102. MARÍN OCETE, A.: El arzobispo don Pedro Guerrero y la politica conciliar española en el siglo XVI, 2 vols., Granada, 1969. REDONDO, A.: «El primer plan sistemático de asimilación de los moriscos granadinos: El doctor Carvajal (1526)», Les morisques et leur temps..., pp. 111-123.

13. Entre los autores que se han ocupado de la acción del Santo oficio en el Reino granadino, destacan los trabajos de: García FuENTES, J. M. ${ }^{a}$ : La Inquisición en Granada en el siglo XVI, Granada, 1981. GARCíA IVARS, F.: La represión del tribunal inquisitorial de Granada (1550-1819), Madrid, 1991. GARRAD, K.: «La Inquisición y los moriscos granadinos (1526-1580)», en Miscelánea de Estudios Árabes y Hebraicos (1960), pp. 55-73. GIL SANJUÁN, J.: «La documentación inquisitorial del tribunal de Granada sobre los moriscos», Religión, identité et sources documentaires sur les morisquues andalous, Tunis, 1984, pp. 257-261. PÉREZ DE COLOSIA RODRIGUEZ, M. I.: «Moriscos sentenciados en los autos de fe del Santo Oficio de Granada: siglo XVI», $V$ Centenaire de la Chute de Grenade, T. II, Túnez, p. 577 y ss. VINCENT, B.: «La Inquisición y los moriscos granadinos», Minorías y Marginados en la España del siglo XVI, Granada, 1990, pp. 119-156.

14. VINCENT, B.: «La Inquisición y los moriscos granadinos», Minorías y Marginados en la España del siglo..., p. 126.

15. SORIA MESA, E.: «De la conquista a la asimilación. La integración de la aristocracia nazarí en la oligarquía granadina. Siglos XV-XVI», Áreas. Revista de Ciencia Sociales, XIV, pp. 49-64. 
local, que pronto adoptarán en todos los terrenos, incluido el religioso, las mismas pautas de comportamiento de sus correligionarios cristianos viejos. Sirva de ejemplo la pertenencia de algunos de sus miembros, como D. Gonzálo Fernández el Zegrí, a una de las cofradías cristiano viejas más importantes de la ciudad, o el encargo por este mismo personaje de un retablo de la "Salutaçion"16.

Por otro lado estimo que sería necesario replantearse la visión de inasimilación que se desprende del texto citado, más aún si tenemos en cuenta que, hasta la fecha, ningún trabajo referente a los conversos granadinos ha entrado a valorar otros testimonios que no hayan sido los oficiales o inquisitoriales con las limitaciones que ello implica. Considero que todo proceso de asimilación es gradual y necesita ser rastreado a través de fuentes que nos faciliten un seguimiento cronológico más o menos continuado. No es un fenómeno homogéneo, por lo que es imprescindible contar con datos que puedan ser agrupados en función de diversas variables: socio-económicas, de género, espaciales, etc. Además si queremos medir el grado de asimilación de los moriscos es imprescindible hacerlo a través de una documentación que nos permita confrontar los comportamientos que estudiamos con los mostrados por aquellos sectores "asimilados" de la sociedad. Sólo así podremos determinar si en realidad, en el momento de la Expulsión, seguían "tan moros" como antes de la Conquista. Afirmación que creo debe ser matizada pues, de ser cierta, ¿cómo interpretar entonces un suceso tan apasionante como las falsificaciones del Sacromonte? El suceso de los libros Plúmbeos está considerado como el último esfuerzo casi patético de los moriscos por "integrarse en la sociedad creada por los Reyes Católicos"17. Fue un intento desesperado "por hispanizar" una parte de la propia cultura con el fin de negociar la pervivencia de algún resto de la misma ${ }^{18}$. Si, como afirma Cabanelas, lo que se pretendía con ellos era dar respuesta "...a unas exigencias que pesaban igualmente sobre vencedores y vencidos, si bien en distinta medida y por razones muy diferentes ${ }^{\prime 19}$, debemos pensar que semejante deseo de aunar intereses y dar respuesta a los mismos, denota ya el deseo de integrarse a una comunidad a la que se sienten vinculados.

La complejidad del problema morisco requiere que intentemos buscar otro enfoque del mismo, en el que dejemos de contemplar al morisco como el proyecto de cristiano que nunca llegó a ser. Es necesario esforzarnos en comprender qué fue para este colectivo ser cristiano, y ser cristiano en una

16. García Pedraza, A.: «La asimilación del morisco Don Gonzalo Fernández el Zegrí: Edición y análisis de su testamento", Al-Qantara, XV (1995), pp. 41-58.

17. HaGerTy, M. J.: Los libros Plúmbeos del Sacromonte, Madrid, 1980, p. 14.

18. García-ARENAL, M.: «El problema morisco...», p. 502.

19. CABANElAS-RodrígueZ, D.: «Intento de supervivencia en el ocaso de una cultura: Los Libros Plúmbeos de Granada", Nueva Revista de Filología Hispánica, T. XXX, 2 (1981), p. 345. 
ciudad que, precisamente por contar con este colectivo, tuvo un clima de espiritualidad especial, aún por estudiar.

Para llevar a cabo este propósito podemos valernos de uno de los recursos más empleados por la historia de las mentalidades: el testamento. El número considerable de escrituras de última voluntad que guarda el archivo notarial de Granada, en comparación a los conservados en otros lugares ${ }^{20}$, nos brinda una oportunidad única para conocer la sensibilidad religiosa del morisco en su nueva fe. A través de ellos podemos rastrear la cordialidad entre el cristiano nuevo y la iglesia e instituciones religiosas de la ciudad, la relación con los vencedores, ahora sus hermanos de fe $\mathrm{y}$, finalmente, de la interpretación que hicieron del modelo cristiano de "buena muerte", con los elementos que del mismo adoptaron junto a las variantes que introdujeron en él. Datos que pueden ayudarnos a determinar en su verdadero sentido el alcance del tan traído y llevado proceso de asimilación.

Aunque en el análisis de su actitud ante la muerte podríamos centrarnos en distintos aspectos, por razones de tiempo y, por ser aún una investigación en proceso de realización, voy a mencionar tan sólo dos: invocaciones y profesiones de fe; y el grado de cordialidad mostrada por este colectivo hacia algunas instituciones religiosas de la ciudad como son las cofradías.

Ilustraremos ambos puntos tomando como referencia los testamentos otorgados entre 1500 y 1526, primeros años de la conversión, y los registrados entre 1560 y 1565, años inmediatamente anteriores a la Guerra.

\section{INVOCACIONES Y PROFESIONES DE FE}

El carácter generalmente estereotipado de estas cláusulas, que en la mayoría de los casos parecen obedecer a los dictados del propio escribano, recomiendan mucha prudencia a la hora de enjuiciarlas. Pero salvando este inconveniente, resultan de gran utilidad para conocer determinadas actitudes religiosas de la sociedad en su conjunto y, lo que es aún más importante, la mayor o menor adecuación de los fieles a los parámetros marcados por la iglesia.

Si comparamos las profesiones de fe de los años sesenta con las dictadas en las dos primeras décadas de la centuria, podemos observar ciertos síntomas de evolución. Evolución que denota la apropiación por parte de los

20. Hemos contabilizado para la primera mitad del siglo XVI, junto a los comprendidos en los años 1555, 1560 y 1565, un total de 295 testamentos de cristianos nuevos. El número es relativamente considerable si tenemos en cuenta que los historiadores que han utilizado este tipo de escritura notarial lo han hecho en una proporción mucho menor. En concreto en la obra ya citada de Tapia Sánchez, se ha consultado 24 escrituras y CARRASCO, R.: «Morisques anciens et morisques nouveaux dans le distric inquisitorial de Cuenca», Mélanges de La Casa de Velázquez, T. XXI (1985), pp. 193-297, utiliza 28 testamentos. 
moriscos de uno de los elementos más característicos de la piedad cristiana: La intervención de María como intermediaria por excelencia entre el hombre y Dios. Entre 1500 y 1526 el formulismo empleado obedeció a un patrón "...rígido, donde no había lugar para devociones particulares y donde incluso las creencias que forman parte del patrimonio religioso popular: la percepción de la Virgen como intermediaria privilegiada, o la repetida alusión a la pasión de Cristo y a los elementos asociados a la misma como el árbol de la Veracruz etc, no se encuentran" 21 . Por el contrario en la década de los sesenta encontramos ya escrituras como la del morisco Daniel Sánchez el Zinety quien alude a María como: "Madre de Nuestro Salvador a quien todos los xpristianos thenemos e ynbocamos por señora e por abogada en nuestras tribulaçiones y nesçesidades" 22 . Si unimos a este hecho las numerosas peticiones que en esta época se hacen de misas en su honor, podemos arriesgarnos a pensar que una de las primeras cosas que asumieron los moriscos de su nueva identidad religiosa fue la figura de María. Su imagen debió ser para ellos el nexo menos extraño entre las creencias pasadas y las que como cristianos se les predicó. No debemos olvidar que en las zonas más islamizadas 23 "veneraban a María, o más bien la defendían y creían en su virginidad..."24.

Pero más interesante aún que la aceptación de su culto, nos parece la adhesión a los dogmas que entorno a su figura defendió la Iglesia. En concreto nos estamos refiriendo a la aceptación de lo que, siglos más tarde, sería el dogma de la Inmaculada. Lorenzo Macaruf alude a "la Ymaculatisima Virgen Santa Maria madre de nuestro Redentor Ihesu Christo" 25 . Su invocación denota no sólo un conocimiento relativamente profundo de los principios defendidos por el catolicismo, sino también una aceptación de las creencias religiosas más caras al pueblo cristiano viejo. Alusión que reviste gran valor si consideramos que a lo largo de estos 64 años sólo existe otra alusión a la Inmaculada: la expresada por la cristiana vieja Ana Castrejón en 1510: "A la Inmaculada Virgen Nuestra Señora... siempre e tenido por particular

21. Garcia PedrazA, A: «Los moriscos ante la muerte: Algunas reflexiones sobre los testamentos otorgados por los moriscos granadinos (1500-1526)», Homenaje a Louis Cardaillac (en prensa).

22. Testamento de Daniel Sánchez el Zinety. A.N.G., Prot. $n^{\circ}$ 137, Alonso Gabano, Luis de Fontiveros, 1564. ff. $1031 \mathrm{r}^{\circ}-1033 \mathrm{r}^{\circ}$.

23. Granada, dada su reciente incorporación a la Corona castellana, era junto a Valencia una de las zonas más islamizadas del país, como lo demuestra el hecho de ser una comunidad arabófona, donde no existe ningún testimonio de literatura aljamiada. Sobre este particular puede consultarse el artículo de MARTíNEZ Ruiz, J.: «Ausencia de literatura Aljamiada y Conservación del Hispano-árabe y de la identidad Arabe-Musulmana en la Granada Morisca (S. XVI)», Chronica Nova, 21 (1993-1994), pp. 405-425.

24. Cardaillac, L.: Moriscos y Cristianos. Un enfrentamiento Polémico (1492-1640), Madrid, 1979.

25. Testamento de Lorenzo Macaruf. A.N.G., Prot. $n^{\circ} 137$, ff. $138 r^{\circ}$. 
abogada" 26 . Este testimonio directo, junto al protagonismo que se le concede a la Inmaculada en los Libros Plúmbeos, nos hace pensar que tal vez María fue uno de los puntos fundamentales en torno a los que el colectivo morisco inicio su "aproximación" a la nueva fe.

\section{COFRADÍAS}

Es en las relaciones moriscos-instituciones religiosas donde hallamos los aspectos más interesantes y, concretamente, en la constatación de moriscos cofrades.

Desde 1492 la religión cristiana se impone en Granada de una manera rotunda, como exigían las circunstancias, utilizando para ello todos los medios disponibles. Se convierte "La palabra y la imprenta, la imagen y el espacio urbano...en medios insustituíbles para ese proceso de evangelización y aculturación" ${ }^{\prime 27}$. Dentro de los medios desplegados por la jerarquía eclesiásti$\mathrm{ca}$, las Cofradías tienen un papel predominante como puntos de atracción y centros de canalización de las manifestaciones religiosas populares. En Granada estas instituciones surgen principalmente en parroquias y conventos, siendo muy escasas las originadas en ermitas u hospitales. En el siglo XVI las cofradías más dinámicas serán las residentes en los conventos.

Centrándonos en el binomio moriscos-cofradías, tenemos las recomendaciones que Fray Hernado de Talavera les dio para que se unieran en ellas "como tienen los cristianos para ayudar de ellas en muerte y en vida" 28 . Por referencias bibliográficas sabiamos de la existencia de dos cofradías moriscas: La Resurrección (masculina), y la Concepçión de Nuestra Señora (femenina), organizada ésta en tiempos del arzobispo don Pedro Guerrero ${ }^{29}$. Pero como

26. Testamento de Ana Castrejón. A.N.G., Prot. de Juan de Rael, 1504-1510, sin foliar.

27. López MuÑoz, M. L.: Las cofradías de la parroquia de Santa María Magdalena de Granada en los siglos XVII-XVIII, Granada, 1992, p. 16.

28. Esta recomendación se encuentra inserta en el Memorial que, en los primeros años de las Conversiones, dirigió el Arzobispo granadino a los moriscos. Gracias a él podemos reconstruir lo que la iglesia esperaba que los moriscos hicieran "como buenos cristianos". Por lo que respecta al tema que nos ocupa -la actitud ante la muerte-, Talavera concreta "La summa de los que querríamos que guardáses" en: "A) Que luego que enfermaren, resçiban los sacramentos de penitencia y de comunión, y en artículo de muerte la extramunción. B) Que hagan los testamentos y obras pías como católicos cristianos, y que sean y seáis sepultados en cementerios bendecidos cerca de vuestras iglesias, según lo hacen los cristianos de nación. C) Que tengáis cofradías...". Una transcripción completa de las Instrucciones del Arzobispo Talavera puede encontrarse en la ya mencionada obra de Gallego y Burín y Gamir Sandoval.

29. La cofradía de la Resurrección es mencionada en la obra de ANTOLínEZ DE BURGOS, J.: Historia Eclesiástica de Granada. Ms. Biblioteca Universidad de Granada, siglo XVIII. La cofradía de la Concepción es reseñada por SANTIBÁÑEZ, J. de: Historia de la Provincia de la Compañía de Jesús, 1648. 
señalaba Garrido Aranda "Aquí acaban nuestras informaciones sobre las cofradías granadinas" 30 . Ahora, basándonos en algunos datos obtenidos en los testamentos, podemos conocer algo más sobre la realidad del morisco cofrade, punto de vital importancia para calibrar las religiosidad de este colectivo.

La cofradía de la Resurrección debió comenzar su bagaje en los primeros meses de 1563, en concreto entre enero y mayo de ese año. En el testamento de Françisco el Caçar, realizado el 10 de enero de 1563 se dice: "Yten mando que aconpañe mi cuerpo la cofradía del Ospital del Albayzin de Granada sy al tienpo de mi fallesçimiento estuviere fundada donde no que aconpañe mi cuerpo la cofradía y hermandad del Santisimo Sacramentos de la yglesia de Sant Salvador"31. En mayo de ese mismo año poseemos documentación que nos la muestra funcionando ya como hermandad. En 1564 estaría plenamente consolidada bajo el nombre de la Santa Resurrección de Nuestro Redentor Thesucristo, como lo avala el testimonio de Lázaro Hernández Albeytar: "Yten mando que el dicho dia de mi enterramiento aconpañe mi cuerpo la cofradía y hermandad del Ospital que nuevamente se a fecho y fundado en el Albayzin de esta çibdad de Granada que se nombra el dicho Ospital de la Santa Resurrección de Nuestro Redentor Ihesucristo $^{\prime \prime 32}$.

Pero al margen de estos datos, poco más sabemos de esta cofradía y del hospital que regentó. Parece ser que el hospital estuvo situado en la Plaza de Bibalbonud, aunque algunos autores lo ubican en la placeta del Abad, al lado sur de la iglesia de S. Nicolás ${ }^{33}$. Era financiado por los moriscos y en él se curaban todas las enfermedades. En 1568, tras el levantamiento, se dio a la ciudad y en 1603 se entregó a Antonio de Aróstegui para la fundación de los Agustinos Descalzos. Como cualquier hospital dependiente de una cofradía se encontraba gobernado por sus propios cofrades, quienes nombraban al administrador o mayordomo. La financiación, en base a rentas y limosnas, iba encaminada a la labor sanitaria, celebraciones religiosas, obras y reparos, como pone de manifiesto la manda que Gonzálo Fernández el Comaxaxa le deja para: "ayuda a los gastos e benefiçio de los pobres del dicho Ospital e...por que los dichos cofrades tengan cuydado de rogar a Dios por mi anima"34.

Podemos afirmar, basándonos en los datos aportados por los testamentos, que la relación entre los moriscos y la cofradía de la Resurrección

30. Garrido Aranda, A.: «Papel de la Iglesia de Granada en la Asimilación de ...», p. 72.

31. Testamento de Francisco el Caçar. A.N.G., Prot. $n^{\circ} 137$, Alonso Gabano, Luis de Fontiveros, 1564 , ff. $50 \mathrm{v}^{\circ}-53 \mathrm{r}^{\circ}$.

32. Testamento de Lázaro Hernández Albeytar, realizado el 25 de noviembre de 1564. A.N.G., Prot. $n^{\circ} 137$, ff. $1675 v^{\circ}-1678 r^{\circ}$.

33. LOPEZ GUZMÁN, R.: Tradición y clasicismo en la Granada del siglo XVI. Arquitectura civil y urbanismo, Granada, 1987, p. 628.

34. Testamento de Gonzalo Fernández el Comaxaxa. A.N.G., Prot. n 137 , ff. $1209 \mathrm{r}^{\circ}-1219 \mathrm{v}^{\circ}$. 
no difirió de la que mantenían los cristianos viejos con estas instituciones piadosas:

- En ambos colectivos el dinero que se designa a la cofradía en las mandas va destinado principalmente a sufragar los gastos ocasionados por la cera: "Yten mando que el dia de my enterramiento aconpañen mi cuerpo la cofradía y hermandad del Santisimo Sacramento de la dicha yglesia de Sant Salvador con su çera e paño que para ello tienen diputado y les pague de mis bienes por el dicho aconpañamiento la limosna acostunbrada para la çera de la dicha cofradia" ${ }^{\prime 35}$.

- Lo que se solicita de ellas es su participación en el entierro y la celebración de sufragios por el alma del otorgante. Veamos como ejemplo la petición de Alonso de Herrera el Hadid: "mando que el dia de mi enterramiento aconpañe mi cuerpo la cofradía y hermandad del Santisimo Sacramento de la dicha yglesia de Sant Salbador de donde yo soi hermano...e les encargo me hagan dezir las misas que son obligados a dezir como a tal hermano que soy de la dicha cofradia"36.

- Los moriscos, igual que los cristianos viejos, dan muestras en sus testamentos de conocer perfectamente el funcionamiento interno de su cofradía, mostrando ambos además un especial interés por saldar las deudas que tuvieran pendientes con ella: "yten mando que el dicho dia de mi enterramiento aconpañe así mismo mi cuerpo la cofradía del Santisimo Sacramento de la dicha yglesia de San Salvador de donde soy cofrade e con su çera como es costumbre e los dichos cofrades son obligados a lo asy faser e mando que se de a la dicha cofradía para ayuda a la çera de ella vn ducado en limosna e se bea lo que yo devo de luminarias e se le pague aquello que paresçiere que devo de las dicha luminarias por el libro de la dicha cofradía"37.

Pero de los testimonios aportados lo más significativo es la constatación de dos hechos:

- Que el binomio moriscos-cofradía no se suscribió exclusivamente a la Cofradía de la Resurrección, sino que se hizo extensible a otras Cofradías, como lo demuestra Lorenzo Albeytar: "yten mando que el dicho dia de my enterramiento aconpañen mi cuerpo la cofradía y hermandad del Santísimo Sacramento de la dicha yglesia de Sant Pablo y San Pedro con su çera y paño que para ello tiene diputado y se les de de limosna para la çera de la dicha cofradía vn ducado e por que yo soy hermano y cofrade de la dicha cofradía

35. Testamento de Elvira Hazbula, realizado el 4 de agosto de 1565. A.N.G., Prot. $\mathrm{n}^{\circ} 137$, ff. $1226 \mathrm{r}^{\circ}-1229 \mathrm{r}^{\circ}$.

36. Testamento de Alonso de Herrera el Hadid, otorgado el 26 de julio de 1563. A.N.G., Prot. $n^{\circ}$ 137, ff. $879 \mathrm{r}^{\circ}-871 \mathrm{r}^{\circ}$.

37. Testamento de Gonzalo Fernández el Comaxaxa, fechado el 30 de julio de 1665. A.N.G., Prot. $\mathrm{n}^{\circ} 137$, ff. $1209 \mathrm{r}^{\circ}-1219 \mathrm{v}^{\circ}$. 
encargo a los ofiçiales y hermanos de ella que con toda brevedad me hagan dezir las misas que son obligados como a tal hermano"38. Esto nos demuestra que los moriscos buscaron legitimar su identidad y reforzar su sociabilidad no sólo en espacios exclusivamente concebidos para ellos, caso de la Cofradía de la Resurrección, sino también a través de la parroquia, institución abierta donde ambas comunidades se encontraban representadas.

- Que igual que entre los cristianos viejos, se daban casos de pertenencia a más de una cofradía. Por ejemplo Lorenzo Albeytar era hermano de la cofradía de la Resurrección y de la Sacramental de su parroquia. Pauta de comportamiento característica de los sectores creyentes más piadosos.

La aparición de moriscos cofrades, dato hasta ahora no constatado, debe ponerse en relación directa con el proceso de cristianización desarrollado en la Ciudad, y la incidencia que éste tuvo sobre el colectivo morisco. Proceso que no puede quedar reducido a un estudio de las conversiones masivas de 1500-1501 y al fracaso de las mismas, cuyo epílogo fue la expulsión definitiva en el siglo XVII. Entre ambas fechas queda todo un periodo por investigar $\mathrm{y}$, sobre todo, resta el seguimiento cronológico del colectivo morisco, de la religiosidad del mismo, analizada no de forma aislada, sino enmarcada en lo que fue el mundo espiritual de la Granada renacentista. Debemos escuchar más que la voz morisca o la voz cristiana, las voces de moriscos y las voces de cristianos.

38. Testamento de Lorenzo Hernández Albeytar, ya citado. Por lo que respecta a la cofradía sacramental de San Pedro y San Pablo sólo sabemos que "aparece mencionada por Henríquez... con origen en el siglo XVI. Era hermandad sacramental de carácter abierto". Tesis doctoral leída en la Universidad de Granada, durante el curso 1991-1992. LópEZ Muñoz, M. L.: Contrarreforma y Cofradías en Granada. Aproximación a la historia de las Cofradías y hermandades de la ciudad de Granada durante los siglos XVII y XVIII. 